\title{
milliPillar: a platform for the generation and real-time assessment of human engineered cardiac tissues
}

Manuel Alejandro Tamargo ${ }^{1,2+}$, Trevor Ray Nash ${ }^{1,2+}$, Sharon Fleischer ${ }^{1+}$, Youngbin Kim $^{l}$, Olaia Fernandez

Vila $^{1,3}$, Keith Yeager ${ }^{1}$, Max Summers ${ }^{1}$, Yimu Zhao ${ }^{1}$, Roberta Lock ${ }^{1}$, Miguel Chavez ${ }^{1}$, Troy Costa ${ }^{1}$ and

Gordana Vunjak-Novakovic ${ }^{1,2 *}$.

\section{Supporting Information}

Figure S1. milliPillar molds.

Figure S2. Preparing the milliPillar reactor.

Figure S3. Mechanical testing of milliPillar PDMS pillars.

Figure S4. Alignment and ventricular characterization of milliPillar tissues cultured for 100 days.

Figure S5. Detailed schematic of functional outputs from custom milliPillar software.

Figure S6. Detailed schematic of ET/MCR stimulation regimen.

Table S1. Comparison of design principles and properties of engineered heart tissue models.

Video S1. Spontaneously beating BS2 milliPillar tissue with NHDFs

Video S2. WTC1 1 milliPillar tissue paced at $1 \mathrm{~Hz}$ with iPSC-CFs

Video S3. WTC11-GCaMP6f milliPillar tissue paced at $1 \mathrm{~Hz}$ with NHCFs.

Video S4. Pillar tracking of millipillar.

Video S5. milliPillar tissue pre-stimulation.

Video S6. milliPillar tissue post-stimulation.

File S1. milliPillar molds CAD design.

File S2. milliPillar stimulator resources.

File S3. milliPillar analysis software. 


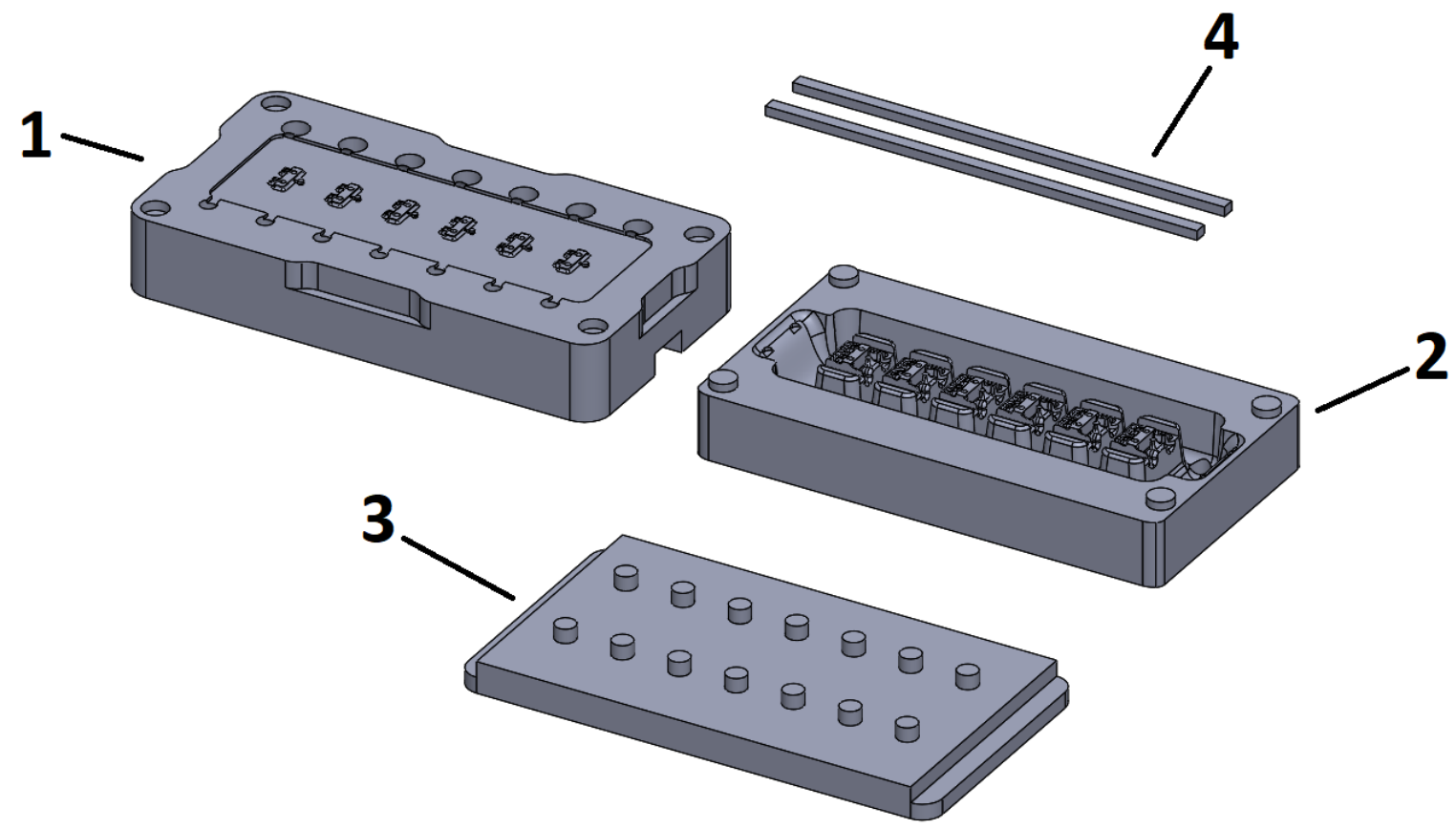

\begin{tabular}{|l|l|}
\hline 1 & Mold top half ("core" with filling and clamping features) \\
\hline 2 & Mold bottom half ("cavity" with holes for casting ejection) \\
\hline 3 & Mold ejection hole plugs \\
\hline 4 & Carbon (graphite) electrodes \\
\hline
\end{tabular}

Figure S1. milliPillar molds. Visual representation of the different components of the mold as labeled in the CAD file. 

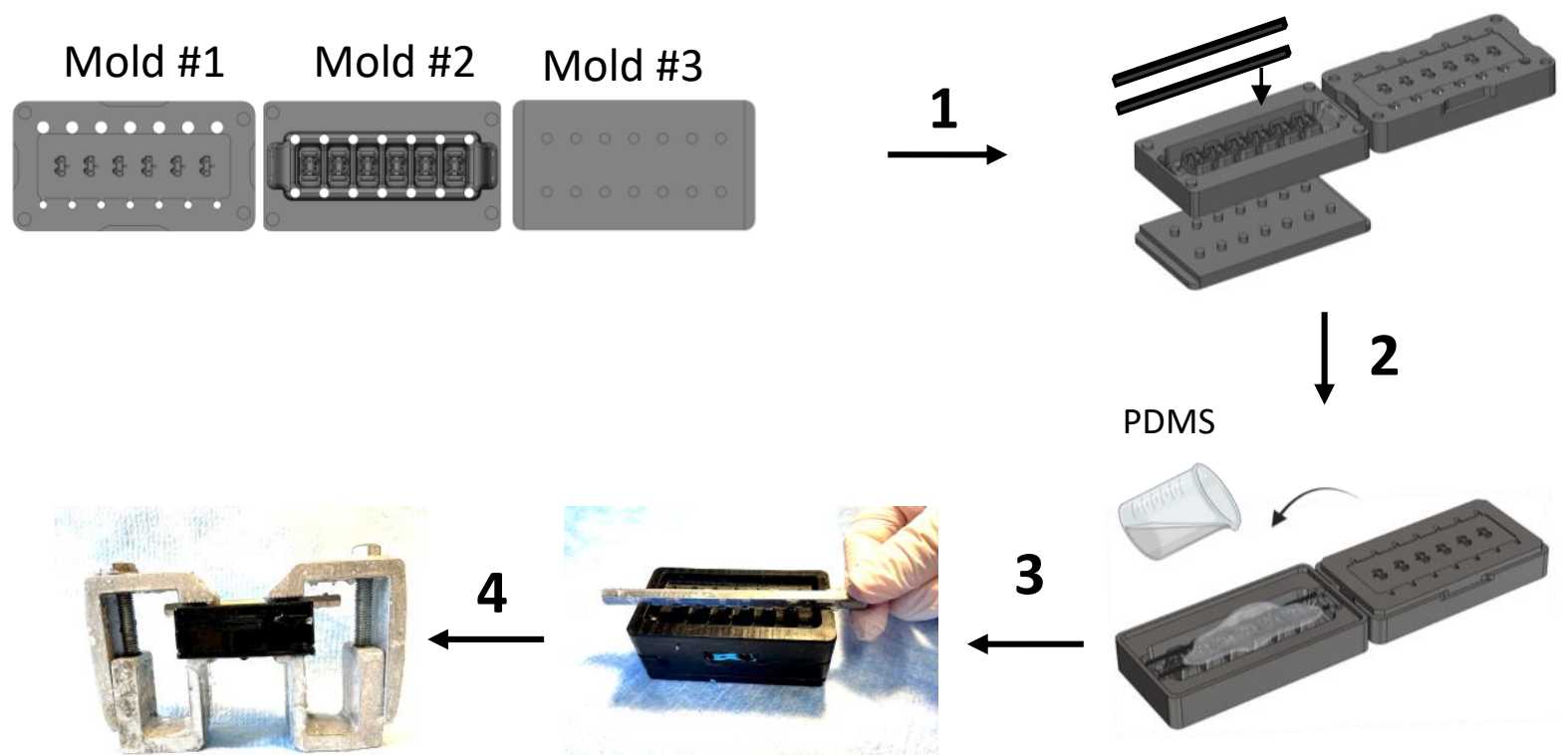

\section{Part 2}

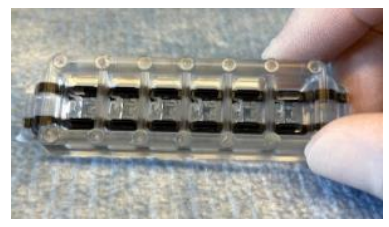

6
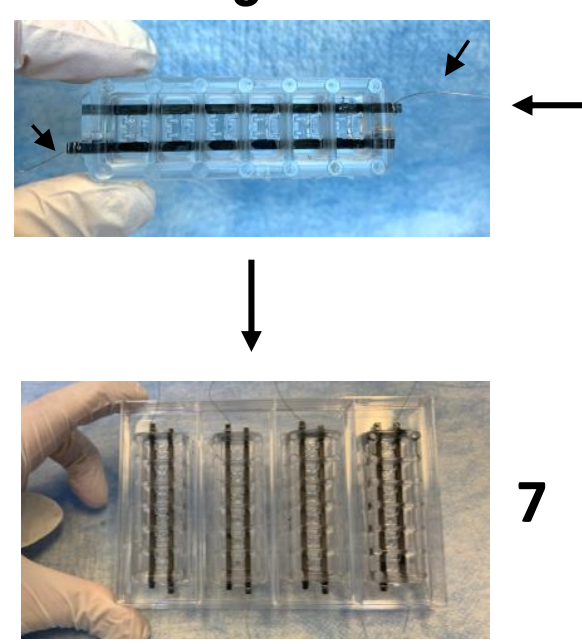

\section{2}
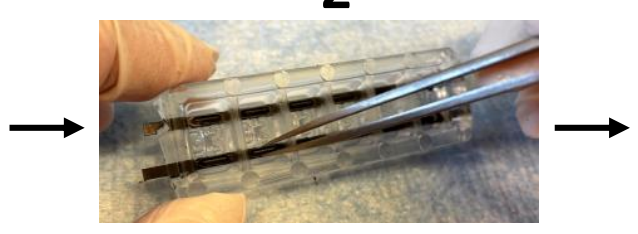

3

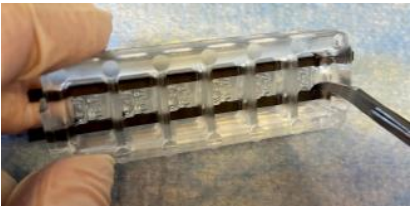

$\downarrow$

Figure S2. Preparing the milliPillar reactor. (A) Simplified graphical protocol for the milliPillar platform fabrication process; Part \#2 and \#3 were assembled and carbon rods were placed into designated inserts in part \#2 (1) Degassed PDMS was used to fill part \#2 and after an additional degassing step, it was topped with part \#3 (2). A Hex screw was added to the top of the mold (3), and two clamps were used to firmly clasp each end of the hex screw (4). (B) Simplified graphical protocol visualizing the PDMS cleanup and carbon rod preparation and exposure. Once the platform was safely excavated from the mold (1), tweezers were used to remove the PDMS tabs to expose electrodes (2). A scalpel was also used to ensure exposure of the inner surface of the rod by scratching off the PDMS film (3). A hole was drilled into the ends of the carbon rod (4). Oxygen plasma treatment was used to bond the bottom of the platform onto a glass slide (5). Platinum wires that connect to the stimulator were tied to the ends of the rods securely (6). Platforms were autoclaved and placed into a 4- well plate. 
A

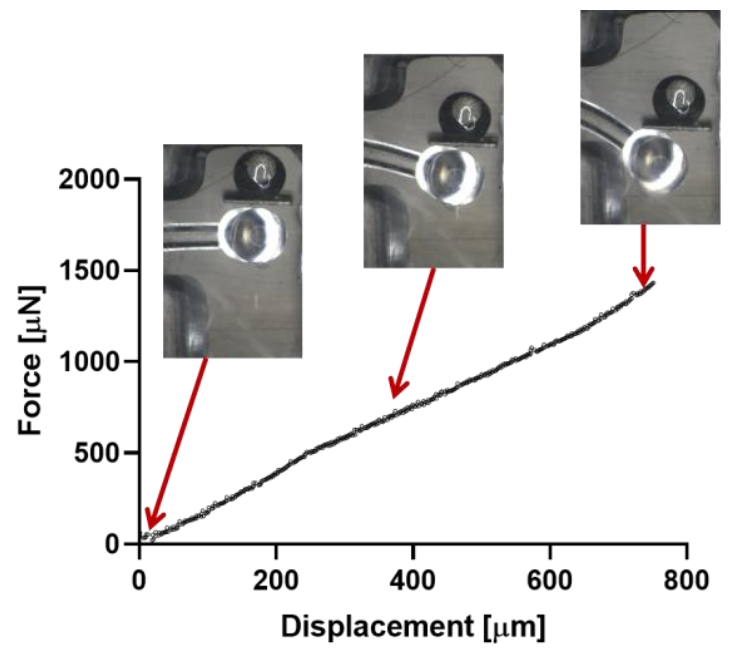

C

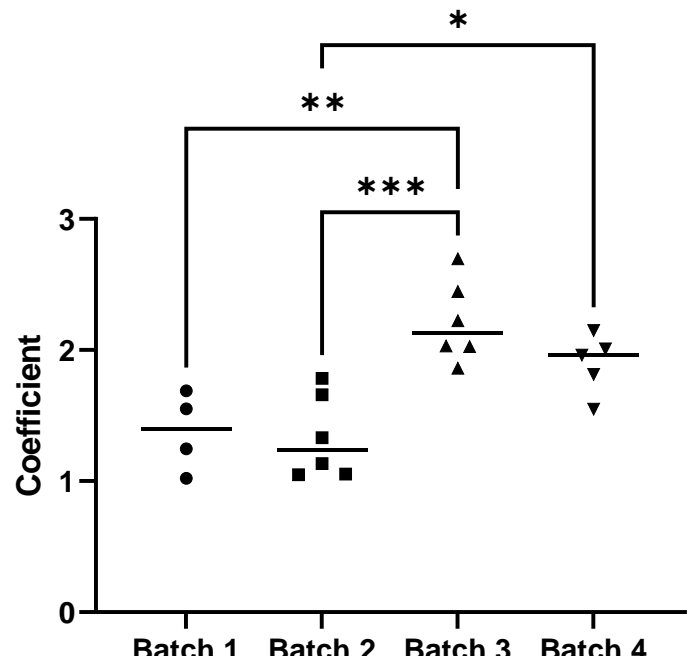

B

\section{Hysteresis}

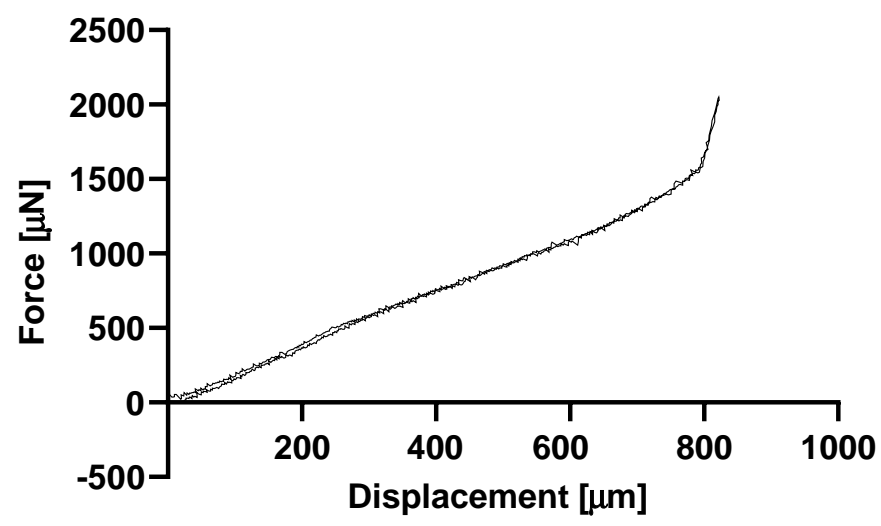

Figure S3: Mechanical testing of milliPillar PDMS pillars. (A) Force as a function of distance coefficients calculated using a microtester. (B)Representative force as a function of distance testing curve showing no apparent hysteresis of PDMS pillars. (C) Batch to batch variation among four batches of milliPillar platforms, $n=4-6$. 
A

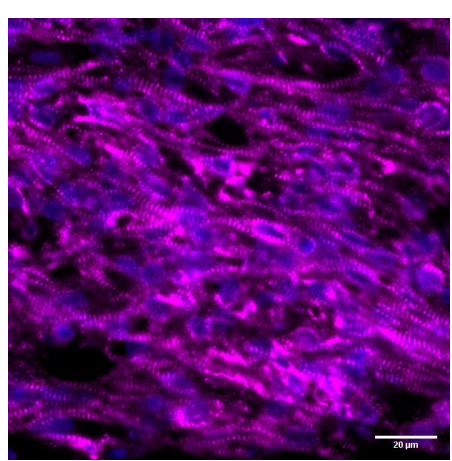

B

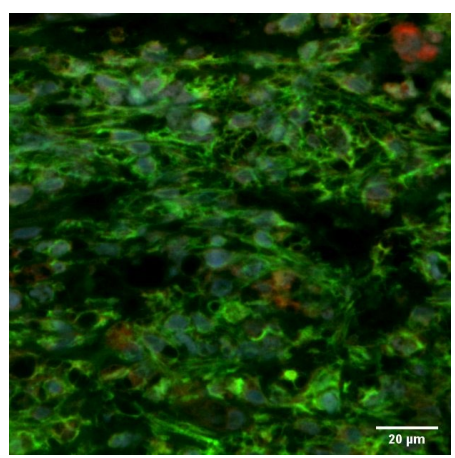

Figure S4. Alignment and ventricular characterization of milliPillar tissues cultured for 100 days.(A) Immunostaining of milliPillar cardiac tissues cultured for 100 days sarcomeric $\alpha$-actinin (magenta).(B) Ventricular characterization of milliPillar cardiac tissue by immunostaining for MLC2v (green), MLC2a (red), DAPI (blue). 


\begin{tabular}{|c|c|}
\hline Feature & Measurements \\
\hline BPM (BF/Cl) & beats per minute \\
\hline Tau $[\mathbf{s}](\mathrm{Cl})$ & $\begin{array}{l}\text { exponential decay constant; time at which the peak intensity is } \\
\text { reduced to } 1 / \mathrm{e} \text { of its maximum value }\end{array}$ \\
\hline Full width half max (FWHM) [s] (Cl) & time between the two $50 \%$ of peak intensity value \\
\hline Full width 90 max (FW90M) [s] (Cl) & time between the two $10 \%$ of peak intensity value \\
\hline Contract $50[\mathrm{~s}](\mathrm{Cl})$ & time between left $50 \%$ intensity to full peak intensity value \\
\hline Relax $50[\mathrm{~s}](\mathrm{Cl})$ & time between full peak to right $50 \%$ intensity value \\
\hline Contract $90[\mathrm{~s}](\mathrm{Cl})$ & time between left $10 \%$ intensity to full peak intensity value \\
\hline Relax $90[\mathrm{~s}](\mathrm{Cl})$ & time between full peak to right $10 \%$ intensity value \\
\hline RR interval $[\mathrm{s}](\mathrm{Cl})$ & average distance between peaks \\
\hline SDRR [s] $(\mathrm{Cl})$ & standard deviation of RR intervals \\
\hline RMSSD [s] (Cl) & $\begin{array}{l}\text { root mean squared successive RR differences (RMS of change in } \\
\text { RR interval) }\end{array}$ \\
\hline $\begin{array}{l}\text { Post Rest Potentiation Force Generation [uN] } \\
\text { (BF) }\end{array}$ & $\begin{array}{l}\text { force generated after } 20 \text { seconds of rest following exertion by } \\
\text { stimulation at } 4 \mathrm{~Hz}\end{array}$ \\
\hline $1 \mathrm{~Hz}$ Force Generation [uN] (BF) & average force generated during stimulation at $1 \mathrm{~Hz}$ \\
\hline Max Beating Frequency $[\mathrm{Hz}](\mathrm{BF})$ & maximum beating frequency, regardless of stimulation frequency \\
\hline Contraction Velocity [ms] (BF) & maximum velocity reached by the tissues during contraction \\
\hline Relaxation Velocity [ms] (BF) & maximum velocity reached by the tissues during relaxation \\
\hline Excitation Threshold (ET) [V] (BF/Cl) & minimum voltage the tissue can capture and respond to at $1 \mathrm{~Hz}$ \\
\hline Maximum Capture Rate (MCR) $[\mathrm{Hz}](\mathrm{BF} / \mathrm{Cl})$ & maximum frequency the tissue can capture and respond to at $5 \mathrm{~V}$ \\
\hline
\end{tabular}

B

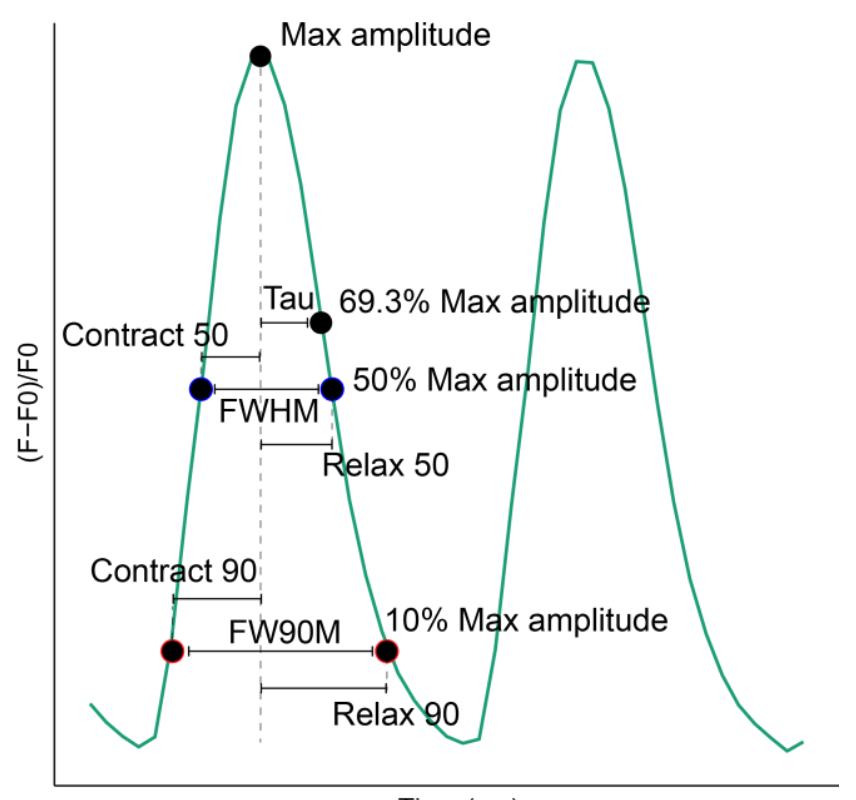

Time (ms)
C
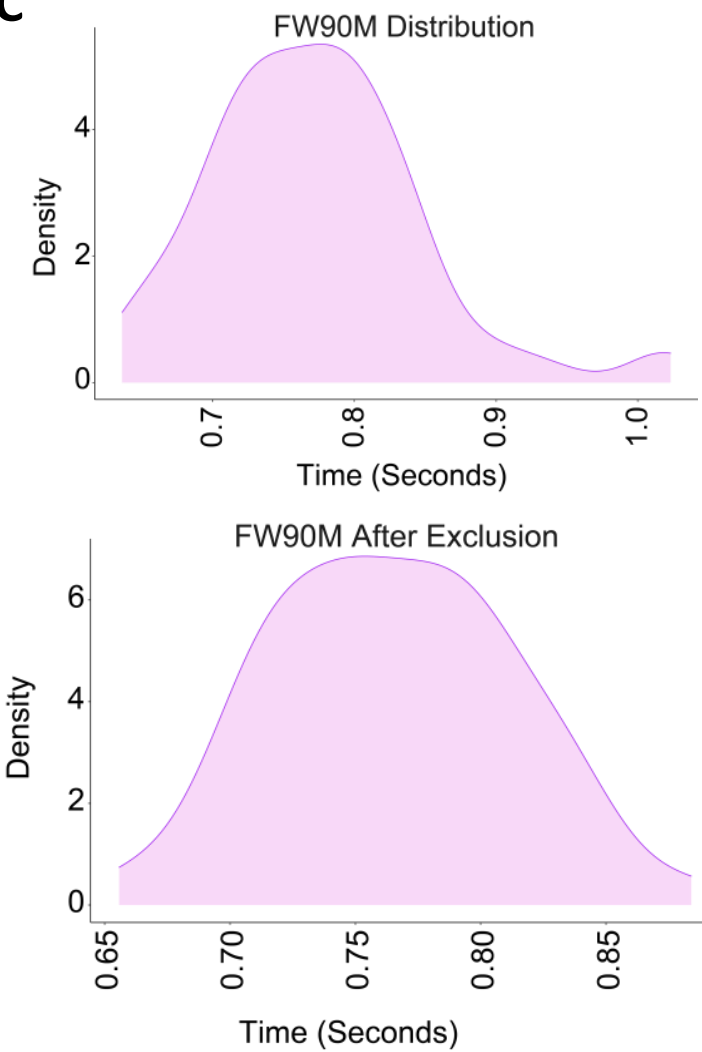

Figure S5. Detailed schematic of functional outputs from custom milliPillar software. (A) Table describing the functional metrics obtained from real-time brightfield and calcium imaging. (B) Schematic of a calcium trace with functional metrics labeled. (C) Representative example of a distribution curve of tissues generated and excluded using FW90M as a quality control metric. The Top $10 \%$ and Bottom $10 \%$ were excluded from this batch to remove outliers and create a normally distributed population. 


\begin{tabular}{|c|c|c|c|c|c|c|c|c|}
\hline Region & Start (s) & Stop (s) & Voltage (V) & Freq (Hz) & Period(s) & First Frame & Last Frame & Duration \\
\hline Spontaneous & 0.00 & 5.00 & 0 & 0 & & 0 & 99 & 100 \\
\hline ET & 5.00 & 10.00 & 5 & 1 & 1.000 & 100 & 199 & 100 \\
\hline ET & 10.00 & 15.00 & 4.5 & 1 & 1.000 & 200 & 299 & 100 \\
\hline ET & 15.00 & 20.00 & 4 & 1 & 1.000 & 300 & 399 & 100 \\
\hline ET & 20.00 & 25.00 & 3.5 & 1 & 1.000 & 400 & 499 & 100 \\
\hline ET & 25.00 & 30.00 & 3 & 1 & 1.000 & 500 & 599 & 100 \\
\hline ET & 30.00 & 35.00 & 2.5 & 1 & 1.000 & 600 & 699 & 100 \\
\hline ET & 35.00 & 40.00 & 2 & 1 & 1.000 & 700 & 799 & 100 \\
\hline ET & 40.00 & 45.00 & 1.5 & 1 & 1.000 & 800 & 899 & 100 \\
\hline ET & 45.00 & 50.00 & 1 & 1 & 1.000 & 900 & 999 & 100 \\
\hline ET & 50.00 & 55.00 & 0.5 & 1 & 1.000 & 1000 & 1099 & 100 \\
\hline FFR & 55.00 & 75.00 & 5 & 0.5 & 2.000 & 1100 & 1499 & 400 \\
\hline FFR & 75.00 & 95.00 & 5 & 1 & 1.000 & 1500 & 1899 & 400 \\
\hline FFR & 95.00 & 115.00 & 5 & 1.5 & 0.667 & 1900 & 2299 & 400 \\
\hline FFR & 115.00 & 135.00 & 5 & 2 & 0.500 & 2300 & 2699 & 400 \\
\hline FFR & 135.00 & 155.00 & 5 & 2.5 & 0.400 & 2700 & 3099 & 400 \\
\hline FFR & 155.00 & 175.00 & 5 & 3 & 0.333 & 3100 & 3499 & 400 \\
\hline FFR & 175.00 & 195.00 & 5 & 3.5 & 0.286 & 3500 & 3899 & 400 \\
\hline FFR & 195.00 & 215.00 & 5 & 4 & 0.250 & 3900 & 4299 & 400 \\
\hline PRP & 215.00 & 235.00 & 0 & 0 & & 4300 & 4699 & 400 \\
\hline PRP & 235.00 & 240.00 & 5 & 1 & 1.000 & 4700 & 4799 & 100 \\
\hline
\end{tabular}

\section{B}
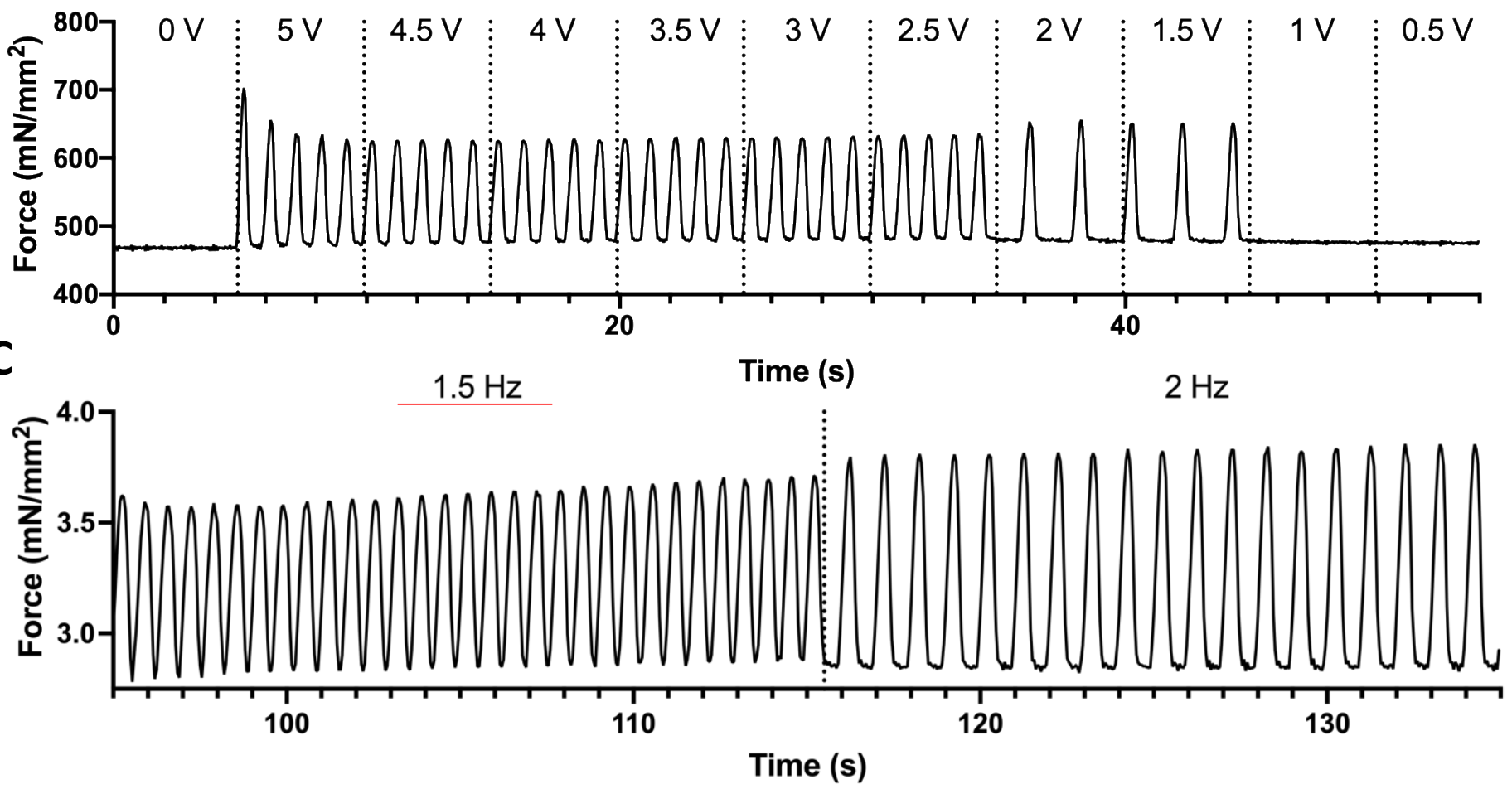

Figure S6. Detailed schematic of ET/MCR stimulation regimen. (A) Table describing the timestamp, voltage, and frequency which tissues are stimulated at during analysis. (B) Schematic of the ET segment of the analysis indicating when the tissue stops responding to the $1 \mathrm{~Hz}$ stimulation (red arrow). (C) Schematic of the MCR segment of the analysis indicating when the tissue stops responding to the stimulation at $5 \mathrm{~V}$ (red underline). 


\begin{tabular}{|c|c|c|c|c|c|c|c|c|c|c|c|}
\hline & $\begin{array}{l}\text { Tissue } \\
\text { Volume }\end{array}$ & $\begin{array}{c}\text { Cells } \\
\text { per } \\
\text { Tissue }\end{array}$ & $\begin{array}{c}\text { Force } \\
\text { Generation } \\
\text { (Measured at 1- } \\
1.5 \mathrm{~Hz} \\
\text { Stimulation) }\end{array}$ & $\begin{array}{c}\text { Well } \\
\text { Volume }\end{array}$ & $\begin{array}{c}\text { Independent } \\
\text { Culture } \\
\text { Wells }\end{array}$ & Throughput & $\begin{array}{l}\text { Charge } \\
\text { Injected } \\
\text { Reported }\end{array}$ & $\begin{array}{c}\text { Electrical } \\
\text { Stimulation } \\
\text { During } \\
\text { Tissue } \\
\text { Culture } \\
\end{array}$ & $\begin{array}{c}\text { Electrical } \\
\text { Stimulator } \\
\text { Commercially } \\
\text { Available }\end{array}$ & $\begin{array}{l}\text { Analysis } \\
\text { Tool in } \\
\text { Public } \\
\text { Repository }\end{array}$ & $\begin{array}{c}\text { Platform } \\
\text { Available in } \\
\text { Public } \\
\text { Repository }\end{array}$ \\
\hline $\begin{array}{l}\text { Turnbull et } \\
\text { al, } 2014\end{array}$ & $100 \mu \mathrm{L}$ & $1 \times 10^{6}$ & $\begin{array}{c}0.57 \pm 0.07 \\
\mathrm{mN} / \mathrm{mm}^{2}\end{array}$ & NA & Yes & Low & No & No & No & No & No \\
\hline $\begin{array}{l}\text { Mannhardt } \\
\text { el al, } 2016\end{array}$ & $100 \mu \mathrm{L}$ & $1 \times 10^{6}$ & $\begin{array}{c}\sim 0.12 \mathrm{mN} \\
\left(0.4 \mathrm{mN} / \mathrm{mm}^{2}\right)^{*}\end{array}$ & $500 \mu \mathrm{L}$ & Yes & Medium & No & No & No & No & No \\
\hline $\begin{array}{l}\text { Jackman et } \\
\text { al, } 2016\end{array}$ & $70 \mu \mathrm{L}$ & $\begin{array}{c}3.75 \times \\
10^{5}\end{array}$ & $\begin{array}{l}23.2 \pm 1.6 \\
\mathrm{mN} / \mathrm{mm}^{2}\end{array}$ & $1.5 \mathrm{~mL}$ & No & Low & No & No & No & No & No \\
\hline $\begin{array}{l}\text { Tiburcy et } \\
\text { al., } 2017\end{array}$ & $500 \mu \mathrm{L}$ & $\begin{array}{l}1 \times 10^{4}- \\
15 \times 10^{6}\end{array}$ & $\begin{array}{l}6.2 \pm 0.8 \\
\mathrm{mN} / \mathrm{mm}^{2}\end{array}$ & NA & No & Low & Yes & No & NA & No & No \\
\hline $\begin{array}{l}\text { Ronaldson- } \\
\text { Bouchard et } \\
\text { al, } 2018\end{array}$ & $200 \mu \mathrm{L}$ & $2 \times 10^{6}$ & $\begin{array}{c}\sim 2.5 \\
\mathrm{mN} / \mathrm{mm}^{2}\end{array}$ & $30 \mathrm{~mL}$ & No & Low & No & Yes & No & Yes & No \\
\hline $\begin{array}{l}\text { Dostanić, } \\
\text { et al } 2019\end{array}$ & $3 \mu \mathrm{L}$ & $4.7 \times 10^{4}$ & $\begin{array}{c}\sim 0.081 \mathrm{mN} \\
\left(0.33 \mathrm{mN} / \mathrm{mm}^{2}\right)^{*}\end{array}$ & $200 \mu \mathrm{L}$ & Yes & High & No & No & No & No & No \\
\hline $\begin{array}{l}\text { Zhao et al, } \\
2020\end{array}$ & $2 \mu \mathrm{L}$ & $1.1 \times 10^{5}$ & $\begin{array}{c}0.051 \pm .025 \\
\mathrm{mN} / \mathrm{mm}^{2}\end{array}$ & NA & No & Low & No & Yes & No & No & No \\
\hline $\begin{array}{l}\text { Thavandiran, } \\
\text { et al, } 2020\end{array}$ & $12 \mu \mathrm{L}$ & $7.5 \times 10^{4}$ & NA & $300 \mu \mathrm{L}$ & Yes & High & No & No & No & No & No \\
\hline $\begin{array}{l}\text { milliPillar, } \\
2021\end{array}$ & $15 \mu \mathrm{L}$ & $5.5 \times 10^{5}$ & $\begin{array}{c}3.22 \pm 0.17 \\
\mathrm{mN} / \mathrm{mm}^{2}\end{array}$ & $400 \mu \mathrm{L}$ & Yes & Medium & Yes & Yes & Open Source & Yes & Yes \\
\hline
\end{tabular}

Table S1. Comparison of design principles and properties of engineered heart tissue models. * Indicates force generation approximated using available information from published data and a cross sectional area calculated by the radius from IHC images. Cross sectional area was calculated by assuming a cylindrical tissue shape $\left(\right.$ Area $\left.=\pi \mathrm{r}^{2}\right)$. 


\section{Supplementary Videos}

\section{Video S1. Spontaneously beating BS2 milliPillar tissue with NHDFs. A}

representative video of a spontaneously beating milliPillar tissue made with 75\% BS2derived cardiomyocytes and 25\% NHDFs. Brightfield videos were recorded for clear visualization of pillar movement and force calculation.

Video S2. WTC11 milliPillar tissue paced at $1 \mathrm{~Hz}$ with iPSC-CFs. A representative video of a milliPillar tissue made with $75 \%$ WTC11-derived cardiomyocytes and $25 \%$ iPSC-derived cardiac fibroblasts. Tissues were stimulated at $1 \mathrm{~Hz}$ using $5 \mathrm{~V} / \mathrm{cm}$ and 20 $\mathrm{nC} / \mathrm{mL}$ charge per pulse for recording. Brightfield videos were recorded for clear visualization of pillar movement and force calculation.

Video S3. WTC11-GCaMP6f milliPillar tissue paced at $1 \mathrm{~Hz}$ with NHCFs. A representative video of a milliPillar tissue made with 75\% WTC11-GCaMP6f-derived cardiomyocytes and 25\% Normal Human Ventricular Cardiac Fibroblasts. Tissues were stimulated at $1 \mathrm{~Hz}$ using $5 \mathrm{~V} / \mathrm{cm}$ and $20 \mathrm{nC} / \mathrm{mL}$ charge per pulse for recording. Brightfield videos were recorded for clear visualization of pillar movement and force calculation.

Video S4. Pillar tracking of milliPillar. Custom analysis software can track the location of the pillar heads throughout the analysis stimulation regimen.

Video S5. milliPillar tissue pre-stimulation. A representative video of a milliPillar tissue 7 days post-fabrication (pre-stimulation) stimulated at $1 \mathrm{~Hz}$ using $5 \mathrm{~V} / \mathrm{cm}$ and 20 $\mathrm{nC} / \mathrm{mL}$ charge per pulse for imaging and analysis. Brightfield videos were recorded for clear visualization of pillar movement.

Video S6. milliPillar tissue post-stimulation. A representative video of a milliPillar tissue 28 days post-fabrication ( 21 days post-stimulation, Ramp protocol) stimulated at $1 \mathrm{~Hz}$ using $5 \mathrm{~V} / \mathrm{cm}$ and $20 \mathrm{nC} / \mathrm{mL}$ charge per pulse for imaging and analysis. Brightfield videos were recorded for clear visualization of pillar movement. 


\section{Supplementary Files}

File S1. milliPillar molds CAD design. A .STEP file that includes the design of the milliPillar Platform

File S2. milliPillar stimulator resources. A .zip file that includes the design and software for the milliPillar Stimulator

File S3. milliPillar analysis software. A .zip file that includes the brightfield and fluorescent calcium analysis software. 